\title{
Role of contact tracing in containing the 2014 Ebola outbreak: a review.
}

\author{
Shrivastava Saurabh, Shrivastava Prateek
}

Shri Sathya Sai Medical College \& Research Institute, Community Medicine

\begin{abstract}
Background: The 2014 outbreak of Ebola virus disease which emerged in the month of March in the year 2014 in Guinea has been declared as a public health emergency of international concern.

Objectives: The objectives of the review article are to assess the role of contact tracing in the Ebola outbreak and to identify the challenges faced by the health workers while performing contact tracing.

Methods: An extensive search of all materials related to the Ebola outbreak and contact tracing was carried out in PubMed, Medline, World Health Organization website and Google Scholar search engines. Keywords used in the search included Ebola virus disease, West-Africa, contact tracing, World Health Organization. Overall 60 articles were selected and included in the discussion.

Results: Contact tracing is an important strategy in epidemiology and refers to the identification and diagnosis of those individuals who have come in contact with an infected person. It ultimately aims to reduce the time span required to detect and treat a case of an infectious disease and hence significantly minimize the risk of transmission to the subsequent susceptible individuals. In-fact, contact tracing continues to remain an important measure, as it aids the epidemiologist in containing the infection.

Conclusion: The strategy of contact tracing has a great potential to significantly reduce the incidence of cases of Ebola virus disease. However, its success is eventually determined by the level of trust between the community and the public health system and the quality of the diagnostic \& treatment services.
\end{abstract}

Keywords: Ebola virus disease, West-Africa, contact tracing, World Health Organization.

DOI: https://dx.doi.org/10.4314/ahs.v17i1.28

Cite as: Saurabh S, Prateek S. Role of contact tracing in containing the 2014 Ebola outbreak: a review. Afri Health Sci. 2017;17(1): 225-236. bttps://dx.doi.org/10.4314/abs.v17i1.28

\section{Introduction}

The 2014 Ebola virus disease (EVD) in Guinea was declared by the World Health Organization (WHO) a public health emergency of international concern in the month of August, 2014. ${ }^{1,2}$ The international stakeholders agreed on this primarily because of its enormous magnitude since its emergence, till the beginning of February 2016, almost 28,639 cases and 11,316 deaths had been reported (total case fatality rate of $39.5 \%$ ) across the ten different nations. ${ }^{3}$ The country-wide estimates suggest that Sierra Leone $(49.3 \%)$ accounted for the maximum global burden, followed by Liberia (37.3\%), while maximum numbers of deaths were reported in Liberia $(42.5 \%)$, and Sierra Leone $(35 \%) .^{3}$

\section{Corresponding author: \\ Shrivastava Saurabh, Shri Sathya Sai Medical \\ College \& Research Institute, \\ Community Medicine \\ Email: prateekbobhate@gmail.com}

African Health Sciences Vol 17 Issue 1, March, 2017
The Ebola epidemic exposed numerous deficiencies in the health care delivery system, and lack of preparedness against a known infectious agent which was initially detected four decades back and since then has appeared around 20 times in different regions of the world. ${ }^{4-6}$ This was really shocking as the current episode of Ebola in West-Africa clearly surpassed the combined estimates of all the previous outbreaks together (both in magnitude and absolute deaths).,5,7 Further, hundreds of the health care professionals also lost their lives while they were extending health care services for the cases of EVD., ${ }^{2,89}$

In-fact, other factors like delayed response by the health and allied sectors towards timely implementation of adequate and appropriate prevention and control measures; poverty and lack of vocational opportunities leading to intensive movement of people across the country borders (playing a significant role in introduction of a new chain of transmission among the inhabitants); shortage of health care personnel and outreach workers; poor awareness among the local residents about the disease / risk factors / mode of transmission / do's and don'ts; development of a sense of fear and mass hysteria among 
people due to the deaths of both people $\&$ health professionals; inclination of people towards traditional healers for their ailments; long existing rituals and traditions which force people to have close contact with deceased at times of their funeral; climate of the affected regions which makes it extremely difficult to use personal protective measures continuously and consistently; and absence of an effective vaccine / drug which can prevent the acquisition of the infection, have together played a massive role in increasing the case load in the affected regions. . $^{1,4,10-14}$

Even now, the disease possesses the potential to spread to different parts of the world because of the existing weaknesses in the international health regulations and migration of large number of people from infected regions in other parts of the world due to varied reasons. ${ }^{15,16} \mathrm{Al}-$ though, currently a significant improvement has been made in the containment of the outbreak in diversified settings, thanks to the efforts of the WHO and other stakeholders, nevertheless in the absence of an effective vaccine, the case load can be only reduced if each of the diagnosed cases is appropriately managed and contacts are followed-up for the maximum incubation period. ${ }^{17-19}$ Contact tracing in the Ebola epidemic is defined as the systematic process of identification, assessment, and management of people who have been exposed to Ebola virus to prevent further transmission of the agent. ${ }^{20,21}$ Contact tracing was used as one of the strategies to control further spread of the Ebola virus but the role of this strategy remains poorly understood. ${ }^{20,21}$ Even though, contact tracing is a critical element in containing the EVD outbreak, nevertheless it is just one of the multi-pronged strategies which are essential to halt the rise in number of cases. $^{22-25}$

\section{Contact tracing in Ebola: an overview}

Contact tracing in the Ebola epidemic is defined as the systematic process of identification, assessment, and management of people who have been exposed to Ebola virus to prevent further transmission of the agent. ${ }^{20,21} \mathrm{~A}$ contact is someone who gives a positive history of exposure to a suspected, probable, or confirmed case of EVD by sleeping in the same household as a case, direct physical contact with the case during the illness or deceased case at funeral/burial preparation rituals, contact with blood or body fluids or clothes or linens of a case, and a baby who has been breastfed by the case. ${ }^{20,22}$
Even though, contact tracing is a critical element in containing the EVD outbreak, it is just one of the multipronged strategies which are essential to halt the rise in number of cases. $^{22-25}$ Contact tracing is closely linked with case detection and investigation processes so that subsequent symptomatic patients can be effectively managed. ${ }^{25,26}$ The exposed persons are followed for a period of 21 days (the maximum incubation period for the disease) from the date of the most recent exposure, so that any symptomatic person can be detected and managed at the earliest, and thus any possibility of subsequent transmission of the virus can be neutralized. ${ }^{22,27}$ Further, as this enables prompt isolation of symptomatic person in a health center, the treatment can be initiated earlier and thus death rates can be minimized. ${ }^{28,29}$

Owing to the enormous burden, long duration, and complex nature of the current EVD outbreak, it has become imperative to implement effective containment measures. ${ }^{28,30}$ Further, it is quite essential to realize that the largest risk of acquiring the Ebola infection is not from confirmed patients, but from the late detection or isolation of the suspect / probable cases. ${ }^{20}$ The approach of contact tracing in the control of EVD epidemic is completely justifiable as almost all of the new cases of Ebola acquire the infection from a contact that has been earlier exposed to a case of EVD. ${ }^{20,30}$ Any person with EVD can begin to transmit the disease to others on appearance of first symptoms, and thus it is crucial to identify and isolate symptomatic patients. ${ }^{19}$ In-fact, contact tracing has been also advocated for the travelers who have been exposed to a suspected or confirmed case of Ebola. ${ }^{22,26}$

However, interruption in the EVD transmission through contact tracing can only be ensured if it is promptly implemented upon identification of any type of EVD case and no time is wasted for the laboratory confirmation of the disease. ${ }^{20,32}$ In general, contact tracing consists of three elements, namely, identification, listing, and subsequently their follow-up. ${ }^{21}$ In the first stage, repeated interviews are conducted with cases of EVD to ascertain all possible contacts. ${ }^{22,33}$ This is essential as most of the individuals after knowing that they are suffering from EVD might not recall all possible contacts due to fear / anxiety, and hence extending psychosocial support is a must. ${ }^{25,34}$

The second stage is of contact listing in which desired information (viz contact's relation to the case, last interac- 
tion, type of interaction, telephone number, etc.) from all the contacts is collected. ${ }^{20}$ At this stage, on confirmation of being a contact, they are informed of their risk status with empathy, signs/symptoms of EVD, preventive measures, and the plan of action for further follow-up. ${ }^{22}$ In order to motivate them, the contacts can be told about the various merits of being a contact as they have prompt access to quality-assured health care services, their other family members can be protected from acquiring the disease, and that they can play a significant role in interrupting the chain of transmission in their locality. ${ }^{30,31}$

Contact listing is followed by the final stage of contact follow-up with the help of a specified follow-up team through daily visits at a pre-defined location and time for a period of 21 days. ${ }^{21}$ If a single team follows-up the contact for the full duration, not only will it facilitate development of trust, but even encourage contacts to report their symptoms, if they develop. ${ }^{22}$ Every attempt should be made to locate the contacts if they are not found for whatsoever reason. ${ }^{22}$ The assistance from the local community leaders can be obtained for those contacts that are not willing to follow-up. ${ }^{31}$ From the health workers' perspective who is engaged in contact tracing, it is strongly advocated that they should adhere to appropriate preventive measures (viz avoiding handshake, maintaining a distance in excess of one meter during interview sessions, using alcohol-based hand rub solutions regularly, not measuring the temperature of the contacts, etc.), in order to prevent the acquisition of infection. ${ }^{21,35}$

However, if there are any issues with the health status of the contact during the follow-up period, they should be evaluated whether they can be categorized as EVD suspects. ${ }^{20,24}$ All of the suspect EVD cases should be immediately isolated in an earmarked unit and subjected to confirmatory laboratory tests (RT-PCR assay for EVD). ${ }^{36}$ If these suspected EVD cases are diagnosed as EVD negative after 3 days of the appearance of symptoms, they can return home for the completion of follow-up. ${ }^{22}$ Further, there is an extensive need to create awareness among the members of community that all those who have been discharged either from the isolation unit or from the follow-up process no longer serve as a threat to the family members or other residents and hence should not be stigmatized. ${ }^{20,22}$ At the same time, any contact that is re-exposed to another case of EVD must undergo a cycle of 21 days of follow-up from the last date of their most recent exposure..$^{21,26}$

The objectives of the review article were to assess the role of contact tracing in the Ebola outbreak and to identify the challenges faced by the health workers while performing contact tracing.

\section{Methodology}

An extensive search of all materials related to the topic was done for four months (September - December 2015) in Pubmed, Medline, WHO website and Google Scholar search engines. Relevant documents, reports, recommendations, guidelines and research articles focusing on the different aspects of Ebola outbreak and contact tracing, published in the period 2004-2015 were included in the review.

\section{Selection of studies}

A total of 69 studies / articles performed with an objective similar to the current were identified initially, of which, nine were excluded due to the unavailability of the complete version of the articles. Overall 60 articles were selected based upon the suitability with the current review objectives and analyzed. Some of them have been mentioned in Table 1

These identified articles, technical reports, review articles, and other forms of research articles were then re-grouped into different sections, namely overview of the utility of contact tracing in the Ebola epidemic, importance of contact tracing in infectious disease control, challenges posed for contact tracers, and lessons learnt in contact tracing. Keywords used in the search comprised of Ebola virus disease, West-Africa, contact tracing, World Health Organization. 
Table 1: Selected research articles

\begin{tabular}{|c|c|c|c|c|}
\hline Author & $\begin{array}{l}\text { Category of } \\
\text { article }\end{array}$ & $\begin{array}{c}\text { Year of } \\
\text { Publication }\end{array}$ & Web link & Reference Citation \\
\hline $\begin{array}{l}\text { Shrivastava } \\
\text { SR, } \\
\text { Shrivastava } \\
\text { PS, } \\
\text { Ramasamy J. }\end{array}$ & $\begin{array}{l}\text { Review } \\
\text { Article }\end{array}$ & 2015 & $\begin{array}{l}\frac{\text { http://www.sciencedirect.com/s }}{\text { cience/article/pii/S2222180814 }} \\
\underline{607799}\end{array}$ & $\begin{array}{l}\text { Shrivastava SR, Shrivastava PS, } \\
\text { Ramasamy J. Ebola disease: an } \\
\text { international public health emergency. } \\
\text { Asian Pac J Trop Dis. 2015; 5(4): 253- } \\
262 .\end{array}$ \\
\hline Chan M. & Perspective & 2014 & $\begin{array}{l}\text { http://www.nejm.org/doi/full/1 } \\
\underline{0.1056 / \text { NEJMp1409859 }}\end{array}$ & $\begin{array}{l}\text { Chan M. Ebola virus disease in West } \\
\text { Africa - no early end to the outbreak. N } \\
\text { Engl J Med. 2014; 371(13): 1183-1185. }\end{array}$ \\
\hline $\begin{array}{l}\text { World Health } \\
\text { Organization. }\end{array}$ & Factsheet & 2015 & $\begin{array}{l}\text { http://www.who.int/mediacentr } \\
\text { e/factsheets/fs103/en/ }\end{array}$ & $\begin{array}{l}\text { World Health Organization. Ebola virus } \\
\text { disease - Fact sheet } N^{\circ} 103 .\end{array}$ \\
\hline Bellizzi S & $\begin{array}{l}\text { Outbreak } \\
\text { News }\end{array}$ & 2014 & $\begin{array}{l}\text { http://www.jidc.org/index.php/j } \\
\text { ournal/article/view/25390049/1 } \\
\underline{\underline{185}}\end{array}$ & $\begin{array}{l}\text { Bellizzi S. The current Ebola outbreak: } \\
\text { old and new contexts. J Infect Dev } \\
\text { Ctries. 2014; 8(11): 1378-8130. }\end{array}$ \\
\hline $\begin{array}{l}\text { Joob B, } \\
\text { Wiwanitkit V. }\end{array}$ & $\begin{array}{l}\text { Letters to } \\
\text { Editor }\end{array}$ & 2014 & $\begin{array}{l}\text { http://www.ncbi.nlm.nih.gov/p } \\
\text { mc/articles/PMC4370092/ }\end{array}$ & $\begin{array}{l}\text { Joob B, Wiwanitkit V. Ebola outbreak in } \\
\text { west Africa. Afr Health Sci. } \\
\text { 2014;14(4):1085. }\end{array}$ \\
\hline $\begin{array}{l}\text { Barry M, } \\
\text { Traoré FA, } \\
\text { Sako FB, } \\
\text { Kpamy DO, } \\
\text { Bah EI, } \\
\text { Poncin M, et } \\
\text { al. }\end{array}$ & $\begin{array}{l}\text { Original } \\
\text { Article }\end{array}$ & 2014 & $\begin{array}{l}\text { http://www.em- } \\
\text { consulte.com/article/941891/al } \\
\text { ertePM }\end{array}$ & $\begin{array}{l}\text { Barry M, Traoré FA, Sako FB, Kpamy DO, } \\
\text { Bah EI, Poncin M, et al. Ebola outbreak } \\
\text { in Conakry, Guinea: epidemiological, } \\
\text { clinical, and outcome features. Med } \\
\text { Mal Infect. 2014; 44(11-12): 491-494. }\end{array}$ \\
\hline
\end{tabular}

\begin{tabular}{|c|c|c|c|c|}
\hline $\begin{array}{l}\text { Victory KR, } \\
\text { Coronado F, } \\
\text { Ifono SO, } \\
\text { Soropogui T, } \\
\text { Dahl BA; } \\
\text { CDC. }\end{array}$ & $\begin{array}{l}\text { Original } \\
\text { Article }\end{array}$ & 2015 & $\begin{array}{l}\text { http://www.cdc.gov/mmwr/pr } \\
\text { eview/mmwrhtml/mm6414a4. } \\
\underline{\text { htm }}\end{array}$ & $\begin{array}{l}\text { Victory KR, Coronado F, Ifono SO, } \\
\text { Soropogui T, Dahl BA; CDC. Ebola } \\
\text { transmission linked to a single } \\
\text { traditional funeral ceremony- } \\
\text { Kissidougou, Guinea, December, 2014- } \\
\text { January 2015. MMWR Morb Mortal } \\
\text { Wkly Rep. 2015; 64(14): 386-388. }\end{array}$ \\
\hline $\begin{array}{l}\text { World Health } \\
\text { Organization. }\end{array}$ & $\begin{array}{l}\text { Technical } \\
\text { Report }\end{array}$ & 2015 & $\begin{array}{l}\text { http://www.who.int/csr/disease } \\
\text { lebola/response/dashboard- } \\
\text { progress-en.pdf?ua=1 }\end{array}$ & $\begin{array}{l}\text { World Health Organization. Ebola } \\
\text { response in action. Geneva: WHO } \\
\text { press; } 2015 .\end{array}$ \\
\hline $\begin{array}{l}\text { World Health } \\
\text { Organization. }\end{array}$ & $\begin{array}{l}\text { Technical } \\
\text { Report }\end{array}$ & 2014 & $\begin{array}{l}\text { http://www.who.int/csr/resourc } \\
\text { es/publications/ebola/contact- } \\
\text { tracing-during-outbreak-of- } \\
\text { ebola.pdf }\end{array}$ & $\begin{array}{l}\text { World Health Organization. Contact } \\
\text { tracing during an outbreak of Ebola } \\
\text { virus disease: Disease surveillance and } \\
\text { response programme area disease } \\
\text { prevention and control cluster. Republic } \\
\text { of Congo: WHO press; } 2014\end{array}$ \\
\hline WHO, CDC. & $\begin{array}{l}\text { Technical } \\
\text { Report }\end{array}$ & 2015 & $\begin{array}{l}\text { http://www.cdc.gov/vhf/ebola/ } \\
\text { pdf/contact-tracing- } \\
\text { guidelines.pdf }\end{array}$ & $\begin{array}{l}\text { WHO, CDC. Implementation and } \\
\text { management of contact tracing for } \\
\text { Ebola virus disease. Geneva: WHO } \\
\text { press; } 2015 .\end{array}$ \\
\hline
\end{tabular}




\begin{tabular}{|c|c|c|c|c|}
\hline $\begin{array}{l}\text { Nyenswah T, } \\
\text { Blackley DJ, } \\
\text { Freeman T, } \\
\text { Lindblade } \\
\text { KA, } \\
\text { Arzoaquoi } \\
\text { SK, Mott JA, } \\
\text { et al. }\end{array}$ & $\begin{array}{l}\text { Weekly } \\
\text { Report }\end{array}$ & 2015 & $\begin{array}{l}\text { http://www.cdc.gov/mmwr/p } \\
\text { review/mmwrhtml/mm6407a } \\
\underline{4 . h t m}\end{array}$ & $\begin{array}{l}\text { Nyenswah T, Blackley DJ, Freeman T, } \\
\text { Lindblade KA, Arzoaquoi SK, Mott JA, } \\
\text { et al. Community quarantine to } \\
\text { interrupt Ebola virus transmission - } \\
\text { Mawah Village, Bong County, Liberia, } \\
\text { August-October, 2014. MMWR Morb } \\
\text { Mortal Wkly Rep. 2015; 64(7): 179- } \\
\text { 182. }\end{array}$ \\
\hline $\begin{array}{l}\text { Gilsdorf A, } \\
\text { Morgan D, } \\
\text { Leitmeyer K. }\end{array}$ & $\begin{array}{l}\text { Review } \\
\text { Article }\end{array}$ & 2012 & $\begin{array}{l}\text { http://www.ncbi.nlm.nih.gov/p } \\
\text { mc/articles/PMC3533809/ }\end{array}$ & $\begin{array}{l}\text { Gilsdorf A, Morgan D, Leitmeyer K. } \\
\text { Guidance for contact tracing of cases } \\
\text { of Lassa fever, Ebola or Marburg } \\
\text { haemorrhagic fever on an airplane: } \\
\text { results of a European expert } \\
\text { consultation. BMC Public Health. } \\
2012 ; 12: 1014 .\end{array}$ \\
\hline $\begin{array}{l}\text { Lopaz MA, } \\
\text { Amela C, } \\
\text { Ordobas M, } \\
\text { Dominguez- } \\
\text { Berjon MF, } \\
\text { Alvarez C, } \\
\text { Martinez M, } \\
\text { et al. }\end{array}$ & $\begin{array}{l}\text { Rapid } \\
\text { Communica } \\
\text { tions }\end{array}$ & 2015 & $\begin{array}{l}\underline{\text { http://www.eurosurveillance.o }} \\
\underline{\text { rg/ViewArticle.aspx?Articleld=2 }} \\
\underline{1003}\end{array}$ & $\begin{array}{l}\text { Lopaz MA, Amela C, Ordobas M, } \\
\text { Dominguez-Berjon MF, Alvarez C, } \\
\text { Martinez M, et al. First secondary case } \\
\text { of Ebola outside Africa: } \\
\text { epidemiological characteristics and } \\
\text { contact monitoring, Spain, September } \\
\text { to November 2014. Euro Surveill. } \\
2015 ; 20(1): 21003 \text {. }\end{array}$ \\
\hline $\begin{array}{l}\text { Smith CL, } \\
\text { Hughes SM, } \\
\text { Karwowski } \\
\text { MP, } \\
\text { Chevalier } \\
\text { MS, Hall E, } \\
\text { loyner SN, et } \\
\text { al. }\end{array}$ & $\begin{array}{l}\text { Original } \\
\text { Article }\end{array}$ & 2015 & $\begin{array}{l}\frac{\text { http://www.cdc.gov/mmwr/pr }}{\text { eview/mmwrhtml/mm6405a2. }} \\
\underline{\text { htm }}\end{array}$ & $\begin{array}{l}\text { Smith CL, Hughes SM, Karwowski MP, } \\
\text { Chevalier MS, Hall E, Joyner SN, et al. } \\
\text { Addressing needs of contacts of Ebola } \\
\text { patients during an investigation of an } \\
\text { Ebola cluster in the United States - } \\
\text { Dallas, Texas, 2014. MMWR Morb } \\
\text { Mortal Wkly Rep. 2015; 64(5): 121- } \\
\text { 123. }\end{array}$ \\
\hline $\begin{array}{l}\text { Nyenswah T, } \\
\text { Fallah M, } \\
\text { Sieh S, Kollie } \\
\text { K, Badio M, } \\
\text { Gray A, et al. }\end{array}$ & $\begin{array}{l}\text { Original } \\
\text { Article }\end{array}$ & 2015 & $\begin{array}{l}\frac{\text { http://www.cdc.gov/mmwr/pre }}{\text { view/mmwrhtml/mm6418a5.ht }} \\
\underline{\mathrm{m}}\end{array}$ & $\begin{array}{l}\text { Nyenswah T, Fallah M, Sieh S, Kollie K, } \\
\text { Badio M, Gray A, et al. Controlling the } \\
\text { last known cluster of Ebola virus } \\
\text { disease - Liberia, January-February } \\
\text { 2015. MMWR Morb Mortal Wkly Rep. } \\
\text { 2015; 64(18): 500-504. }\end{array}$ \\
\hline $\begin{array}{l}\text { Kaasik- } \\
\text { Aaslav K, } \\
\text { Coulombier } \\
\text { D. }\end{array}$ & Editorial & 2015 & $\begin{array}{l}\text { http://www.eurosurveillance.or } \\
\text { g/ViewArticle.aspx?Articleld=21 } \\
\underline{075}\end{array}$ & $\begin{array}{l}\text { Kaasik-Aaslav K, Coulombier D. The tail } \\
\text { of the epidemic and the challenge of } \\
\text { tracing the very last Ebola case. Euro } \\
\text { Surveill. } 2015 ; 20(12): 21075 \text {. }\end{array}$ \\
\hline
\end{tabular}




\begin{tabular}{|c|c|c|c|c|}
\hline $\begin{array}{l}\text { Hagan JE, } \\
\text { Smith W, } \\
\text { Pillai SK, } \\
\text { Yeoman K, } \\
\text { Gupta S, } \\
\text { Neatherlin J, } \\
\text { et al. }\end{array}$ & $\begin{array}{l}\text { Weekly } \\
\text { Report }\end{array}$ & 2015 & $\begin{array}{l}\text { http://www.cdc.gov/mmwr/p } \\
\text { review/mmwrhtml/mm6407a } \\
\text { 5.htm }\end{array}$ & $\begin{array}{l}\text { Hagan JE, Smith W, Pillai SK, Yeoman } \\
\text { K, Gupta S, Neatherlin J, et al. } \\
\text { Implementation of Ebola case-finding } \\
\text { using a village chieftaincy taskforce } \\
\text { in a remote outbreak - Liberia, } 2014 \text {. } \\
\text { MMWR Morb Mortal Wkly Rep. } \\
\text { 2015; 64(7): 183-185. }\end{array}$ \\
\hline $\begin{array}{l}\text { Wells C, } \\
\text { Yamin D, } \\
\text { Ndeffo- } \\
\text { Mbah L, } \\
\text { Wenzel N, } \\
\text { Gaffney G, } \\
\text { Townsend P, } \\
\text { et al. }\end{array}$ & $\begin{array}{l}\text { Original } \\
\text { Article }\end{array}$ & 2015 & $\begin{array}{l}\text { http://www.ncbi.nlm.nih.gov/p } \\
\underline{\text { mc/articles/PMC4449200/ }}\end{array}$ & $\begin{array}{l}\text { Wells C, Yamin D, Ndeffo-Mbah L, } \\
\text { Wenzel N, Gaffney G, Townsend P, et } \\
\text { al. Harnessing case isolation and ring } \\
\text { vaccination to control Ebola. PLoS } \\
\text { NegITrop Dis. 2015; 9(5): e0003794. }\end{array}$ \\
\hline $\begin{array}{l}\text { Logan G, } \\
\text { Vora NM, } \\
\text { Nyensuah } \\
\text { TG, Gasasira } \\
\text { A, Mott J, } \\
\text { Walke H, et } \\
\text { al. }\end{array}$ & $\begin{array}{l}\text { Weekly } \\
\text { Report }\end{array}$ & 2014 & $\begin{array}{l}\text { http://www.cdc.gov/mmwr/pr } \\
\text { eview/mmwrhtml/mm6344a6. } \\
\underline{\text { htm }}\end{array}$ & $\begin{array}{l}\text { Logan G, Vora NM, Nyensuah TG, } \\
\text { Gasasira A, Mott J, Walke H, et al. } \\
\text { Establishment of a community care } \\
\text { center for isolation and management } \\
\text { of Ebola patients - Bomi County, } \\
\text { Liberia, October 2014. MMWR Morb } \\
\text { Mortal Wkly Rep. 2014; 63(44): 1010- } \\
\text { 1012. }\end{array}$ \\
\hline $\begin{array}{l}\text { Tracey LE, } \\
\text { Regan AK, } \\
\text { Armstrong } \\
\text { PK, Dowse } \\
\text { GK, Effler } \\
\text { PV. }\end{array}$ & $\begin{array}{l}\text { Rapid } \\
\text { Communic } \\
\text { ations }\end{array}$ & 2015 & $\begin{array}{l}\frac{\text { http://www.eurosurveillance.o }}{\text { rg/ViewArticle.aspx?Articleld= }} \\
\underline{\underline{20999}}\end{array}$ & $\begin{array}{l}\text { Tracey LE, Regan AK, Armstrong PK, } \\
\text { Dowse GK, Effler PV. EbolaTracks: an } \\
\text { automated SMS system for } \\
\text { monitoring persons potentially } \\
\text { exposed to Ebola virus disease. Euro } \\
\text { Surveill. 2015; 20(1): } 20999 .\end{array}$ \\
\hline
\end{tabular}

\begin{tabular}{|c|c|c|c|c|}
\hline $\begin{array}{l}\text { Gesser- } \\
\text { Edelsburg A, } \\
\text { Shir-Raz Y, } \\
\text { Hayek S, } \\
\text { Sassoni-Bar } \\
\text { Lev } 0 .\end{array}$ & $\begin{array}{l}\text { Original } \\
\text { Article }\end{array}$ & 2015 & $\begin{array}{l}\text { http://www.ajicjournal.org/arti } \\
\text { cle/S0196-6553(15)00150- } \\
\underline{9 / \text { fulltext }}\end{array}$ & $\begin{array}{l}\text { Gesser-Edelsburg A, Shir-Raz Y, Hayek } \\
\text { S, Sassoni-Bar Lev O. What does the } \\
\text { public know about Ebola? The public's } \\
\text { risk perceptions regarding the current } \\
\text { Ebola outbreak in an as-yet unaffected } \\
\text { country. Am J Infect Control. 2015; } \\
\text { 43(7): } 669-675 \text {. }\end{array}$ \\
\hline $\begin{array}{l}\text { Chung WM, } \\
\text { Smith JC, } \\
\text { Weil LM, } \\
\text { Hughes SM, } \\
\text { Joyner SN, } \\
\text { Hall EM, et } \\
\text { al. }\end{array}$ & $\begin{array}{l}\text { Original } \\
\text { Article }\end{array}$ & 2015 & $\begin{array}{l}\text { http://annals.org/article.aspx?ar } \\
\text { ticleid=2297228 }\end{array}$ & $\begin{array}{l}\text { Chung WM, Smith JC, Weil LM, Hughes } \\
\text { SM, Joyner SN, Hall EM, et al. Active } \\
\text { tracing and monitoring of contacts } \\
\text { associated with the first cluster of Ebola } \\
\text { in the United States. Ann Intern Med. } \\
\text { 2015; 163(3): 164-173. }\end{array}$ \\
\hline $\begin{array}{l}\text { World Health } \\
\text { Organization. }\end{array}$ & $\begin{array}{l}\text { Technical } \\
\text { Report }\end{array}$ & 2012 & $\begin{array}{l}\frac{\text { http://apps.who.int/iris/bitstrea }}{\mathrm{m} / 10665 / 75170 / 1 / \text { WHO HSE }} \\
\underline{\text { GCR 2012.13 eng.pdf }}\end{array}$ & $\begin{array}{l}\text { World Health Organization. } \\
\text { Communication for behavioural impact } \\
\text { (COMBI): A toolkit for behavioural and } \\
\text { social communication in outbreak } \\
\text { response. Geneva: WHO press; } 2012 \text {. }\end{array}$ \\
\hline Gulland A. & News & 2014 & $\begin{array}{l}\text { http://www.bmj.com/cgi/pmidl } \\
\text { ookup?view=long\&pmid=25193 } \\
934\end{array}$ & $\begin{array}{l}\text { Gulland A. More health staff are } \\
\text { needed to contain Ebola outbreak, } \\
\text { warns WHO. BMJ. 2014; 349: g5485. }\end{array}$ \\
\hline
\end{tabular}




\begin{tabular}{|c|c|c|c|c|}
\hline $\begin{array}{l}\text { Patel U, } \\
\text { Pharr JR, } \\
\text { Ihesiaba C, } \\
\text { Oduenyi FU, } \\
\text { Hunt AT, } \\
\text { Patel D, et al. }\end{array}$ & $\begin{array}{l}\text { Original } \\
\text { Article }\end{array}$ & 2015 & $\begin{array}{l}\text { http://www.ncbi.nlm.nih.gov/ } \\
\text { pmc/articles/PMC4803986/ }\end{array}$ & $\begin{array}{l}\text { Patel U, Pharr JR, Ihesiaba C, Oduenyi } \\
\text { FU, Hunt AT, Patel D, et al. Ebola } \\
\text { outbreak in Nigeria: Increasing ebola } \\
\text { knowledge of volunteer health } \\
\text { advisors. Glob J Health Sci. 2015; } 8(1) \text { : } \\
46199 .\end{array}$ \\
\hline $\begin{array}{l}\text { Reina-Ortiz } \\
\text { M, Hoare I, } \\
\text { Sharma V, } \\
\text { Izurieta R. }\end{array}$ & Editorial & 2015 & $\begin{array}{l}\text { http://www.ncbi.nlm.nih.gov/p } \\
\text { mc/articles/PMC4448324/ }\end{array}$ & $\begin{array}{l}\text { Reina-Ortiz M, Hoare I, Sharma V, } \\
\text { Izurieta R. State of the globe: Ebola } \\
\text { outbreak in the western world: Are we } \\
\text { really ready? J Glob Infect Dis. 2015; } \\
7(2): 53-55 .\end{array}$ \\
\hline $\begin{array}{l}\text { Matua GA, } \\
\text { Wal DM. }\end{array}$ & $\begin{array}{l}\text { Original } \\
\text { Article }\end{array}$ & 2015 & $\begin{array}{l}\text { http://journals.Iww.com/jnr- } \\
\text { twna/pages/articleviewer.aspx? } \\
\text { year=2015\&issue=09000\&articl } \\
\text { e=00009\&type=abstract }\end{array}$ & $\begin{array}{l}\text { Matua GA, Wal DM. Living under the } \\
\text { constant threat of Ebola: A } \\
\text { phenomenological study of survivors } \\
\text { and family caregivers during an Ebola } \\
\text { outbreak. J Nurs Res. 2015; 23(3): 217- } \\
224 \text {. }\end{array}$ \\
\hline $\begin{array}{l}\text { Wiwanitkit } \\
\text { V, Tambo E, } \\
\text { Ugwu EC, } \\
\text { Ngogang JY, } \\
\text { Zhou XN. }\end{array}$ & $\begin{array}{l}\text { Letters to } \\
\text { Editor }\end{array}$ & 2015 & $\frac{\text { http://www.ncbi.nlm.nih.gov/p }}{\text { mc/articles/PMC4322436/ }}$ & $\begin{array}{l}\text { Wiwanitkit V, Tambo E, Ugwu EC, } \\
\text { Ngogang JY, Zhou XN. Are surveillance } \\
\text { response systems enough to effectively } \\
\text { combat and contain the Ebola } \\
\text { outbreak? Infect Dis Poverty. 2015; } \\
4(1): 7 \text {. }\end{array}$ \\
\hline
\end{tabular}

\section{Results}

The utility of contact tracing in interrupting the chain of transmission can only be achieved, if it is implemented promptly upon identification of an EVD case (including suspected, probable and confirmed), without waiting for laboratory confirmation. On detection of any potential EVD case, an investigation team (team of trained experts with good communication skills and knowledge about socio-cultural norms of the people) should be immediately mobilized to thoroughly assess the case for their complaints, type of exposure, and any predisposing risk factors. If the person does meet the definition of an EVD case, the investigation team should comprehensively interview them $\&$ their family members in a safe $\&$ conducive environment to ascertain all potential contacts since the onset of symptoms. Each of the identified contacts should be assessed individually and explained to about the potential risk, preferably by an epidemiologist. In case, they are having symptoms of the disease, the case management team should be activated and they are managed accordingly, otherwise each one of them should be followed-up for 21 days to look for appearance of Ebola-related clinical features.

In an attempt to interrupt the chain of transmission from the first secondary case of EVD detected in a health care worker in Madrid, Spain, the investigation team identified a total of 232 contacts and followed-them up for 21 days. However, no positive EVD case was detected among these identified contacts. The investigation team employed vehicles as well as mobile phones for implementing contact tracing. ${ }^{27}$

In the Texas state of the United States, the first imported case of the disease was confirmed towards the end of September, 2014, and subsequently two health care workers also acquired the infection. The investigation team identified around 179 contacts (149 health workers +20 
community contacts \{school children, vulnerable people, homeless individual, etc. $\}+10$ persons transported in the same ambulance as that of first EVD case) from these 3 cases. The investigation team employed vehicles \& mobile phones for implementing contact tracing. All these contacts were quarantined in different settings based on their need, while the health workers voluntarily accepted for self-quarantine. ${ }^{28}$

On May 9, 2015, Liberia was declared free of Ebola outbreak by the WHO following the detection of no new case of EVD, the index case for which originated on 29 December 2014. This led to identification of another 21 associated cases. As a part of the containment measure, contact tracing by the member of the investigation team led to the identification of 745 contacts for this cluster over the 6 -week period, including 166 health workers. All these identified contacts were counselled about the potential risk and were followed-up for the next 3 weeks.

In the Kayah region of Liberia, a 48 year old woman died with symptoms of EVD on 21 October 2014. She was buried according to all the traditional practices, which included body contact with the deceased in various ways (grooming, touching, kissing, etc.). Subsequently, in the next 3 weeks, 21 cases of EVD were identified in the five neighboring villages, which were epidemiologically related to the deceased. As the affected region had no significant health infrastructure, assistance was asked from the different stakeholders, which then responded by creating awareness among local people, establishment of a temporary isolation and treatment facility, and contact tracing. The act of contact tracing was quite difficult and challenging as it was found that some of the contacts had fled to the nearby forest, raising a concern of spread of the infection to the neighboring villages as well. Further, there was no restriction on the movement of the people across these villages, and most of them lacked cellular connectivity and this could only be reached by footpaths across the forests. ${ }^{31}$

Owing to all these reasons, it was concluded that the traditional approach of contact tracing will not be effective to interrupt the disease transmission. Thus, a novel active surveillance network was established with the help of the chieftancy task force (comprising of community leaders, plus representatives of men, women, youths, and elders of the community) to ensure village-to-village communication without investing much on resources or training, and at the same time addressing the challenge of lack of means of communication or transportation in the areas. The ultimate aim was to ensure active case finding and reporting of deaths at the village level on every alternate day (even if nil), to the district health team. A simple reporting format was designed and community representatives were oriented about the same. On detection of each probable case, the district health team followed-up each one of them on an emergency basis. In one of the villages one suspected case and one death was reported, both of which were later on confirmation due to non-Ebola reasons. ${ }^{31}$

\section{Challenges encountered}

Even though, the strategy of contact tracing has shown immense potential, but in the early part of the outbreak, it could not be implemented in most of the affected settings, due to enormous caseload and lack of health care staff. ${ }^{37}$ Further, identification of all possible contacts in itself is a logistical challenge as most of them cannot be traced due to the absence of any specific addresses or use of nicknames. ${ }^{20,38}$ Anyways, contact tracing can only deliver positive results if it is promptly started after case finding and the identified cases are efficiently managed as well. ${ }^{39}$ In short, all aspects of the response have to be effectively addressed while preparing for, implementing, and managing contact tracing. ${ }^{21,37-39}$

As already discussed, the output of contact tracing is predominantly determined by the active involvement of the members of the communities. ${ }^{22}$ In-fact, the willingness of the contacts to get enrolled is eventually determined by their level of understanding about EVD, the associated stigma from colleagues / family members / community, and fear of being prohibited from workplace / school. ${ }^{40,41}$ Since the emergence of the recent Ebola outbreak in West Africa, a sense of fear has developed among the local residents and even among the people residing in unaffected regions due to the novelty of exposure to the virus, poor preparedness, and ill-equipped status of the health care delivery system. ${ }^{1,4,42}$ The problem of mass hysteria was further magnified due to the role of media, and incorrect knowledge among the people about the disease or its mode of spread. ${ }^{43}$ Furthermore, myths like people who are listed as contacts are the ones who are more likely to die because of the disease has also interfered with the universal implementation of contact tracing. ${ }^{43,44}$

African Health Sciences Vol 17 Issue 1, March, 2017 
Another pre-requisite for the success of contact tracing is the existence of an accurate and culturally-sensitive communication. ${ }^{40,41,45}$ Any insensitive / ambiguous message or practices during outbreaks can reverse all the achieved gains. ${ }^{45}$ On the contrary, the health sector failed miserably in establishing any sort of trust with the local communities in the affected regions. ${ }^{4,45-47}$ Further, factors like poor laboratory support, and the absence of an earmarked place either to isolate or to administer treatment, never created enough support for the health workers to succeed in developing good relationships with the local community. ${ }^{37,39,44}$

In addition, factors like the need to promptly and comprehensively identify all the contacts, distribution of the cases / contacts in a wide geographical area, ensuring active monitoring of all contacts for three weeks, restricting movements of all the identified contacts, lack of financial assistance, poor accessibility in the affected regions due to resistance from the local residents, and extending humanitarian support services to address the non-clinical needs of contacts, also limited the utility and application of contact tracing in the local community. ${ }^{39,42,46,48}$ As EVD resulted in the deaths of thousands of health workers as well while they were providing health services to the infected cases, a majority of them were also not willing to actively participate in contact tracing. ${ }^{4,47}$ Finally, this study has showed that the Ebola virus tends to persist in semen even after nine months of completion of treatment. ${ }^{49}$ This in itself is a big challenge, as it further emphasizes the importance of contact tracing in containing the outbreak of Ebola virus disease. ${ }^{49}$

\section{Lessons learnt in contact tracing}

Even though, contact tracing could not be successfully implemented in the initial stages of the current outbreak, the disease was contained later on and most of the subsequent new chains of eruption of disease were prevented due to the implementation of contact tracing in all the affected regions. ${ }^{50,51}$ This massive success could be achieved because the international stakeholders have succeeded in achieving the community involvement. ${ }^{52}$ In-fact, confirmatory evidence is available to suggest that contact tracing played a significant role in containing the epidemics of EVD in heterogeneous settings. ${ }^{50,51,53}$ However, it will be wrong to give complete credit to contact tracing, as it was ably supported by improved diagnostic and therapeutic services. ${ }^{54}$
A major credit for the success of contact tracing goes into the strategy which was adopted to communicate the appropriate message, and the psycho-social support offered to the members of the community. ${ }^{55,56}$ The policy makers took appropriate steps to ensure involvement of the community and negate stigma by engaging and educating community leaders about the signs and symptoms of the EVD, its mode of transmission, and steps needed to contain the infection in the community; fostering linkages with religious centers to deliver accurate message to the community; creating awareness among the general population using different modes of mass media communication; offering psychosocial support to constructively deal with the fear associated with EVD; and educating the media to publicise only important issues and at the same time respect the confidentiality for cases and contacts. ${ }^{57-61}$

If the health officials really wish to contain the future outbreaks of EVD, without compromising the lives of thousands of people, there is a great need to be prepared for any such future emergence of the disease. ${ }^{55}$ However, with regard to contact tracing, benefits can only be obtained if there is a mechanism to implement the practice of contact tracing right from the onset of the outbreak..$^{62,63}$ Further, in order to build trust between the health officials and the local community, there is a great need to engage the community stakeholders and even members of the community during the preparation phase so that a sense of ownership can be inculcated among them..$^{55,56}$

In addition, media can be utilized with great effect to discourage any possible risk of stigma associated with case or contacts. ${ }^{57}$ On the contrary, emphasis should be given towards motivating people to give full support to the public health system. ${ }^{4}$ Another effective approach to neutralize the operational constraint of tracking the contacts, innovative approaches like adhering to the global positioning systems or an automated short message services mechanism can be implemented. ${ }^{22,38}$

\section{Conclusion}

The strategy of contact tracing has a great potential to significantly reduce the incidence of cases of Ebola virus disease. However, its success is eventually determined by the level of trust between the community and the public health system and the quality of the diagnostic \& treatment services. 


\section{References}

1. Shrivastava SR, Shrivastava PS, Ramasamy J. Ebola disease: an international public health emergency. Asian Pac J Trop Dis. 2015; 5(4): 253-262.

2. Hawkes N. Ebola outbreak is a public health emergency of international concern, WHO warns. BMJ. 2014; 349: g5089.

3. World Health Organization. Ebola Situation Report -3 February 2016; 2016. Available from: http://apps. who.int/ebola/current-situation/ebola-situation-report-3-february-2016

4. Chan M. Ebola virus disease in West Africa - no early end to the outbreak. N Engl J Med. 2014; 371(13): 11831185 .

5. Centers for Disease Control and Prevention. Outbreaks chronology: Ebola virus disease; 2014. Available from: http://www.cdc.gov/vhf/Ebola/outbreaks/history/chronology.html

6. Goozner M. History and the Ebola outbreak. Mod Healthc. 2014; 44(33): 24.

7. World Health Organization. Ebola virus disease - Fact sheet N¹03; 2015. Available from: http://www.who.int/ mediacentre/factsheets/fs103/en/

8. Stingl P. Ebola outbreak in West-Africa. MMW Fortschr Med. 2015; 157(14): 50-53.

9. Bellizzi S. The current Ebola outbreak: old and new contexts. J Infect Dev Ctries. 2014; 8(11): 1378-8130.

10. Gursky EA. Rising to the challenge: The Ebola outbreak in Sierra Leone and how Insights into one nongovernmental organization's response can inform future core competencies. Disaster Med Public Health Prep. 2015; 9(5): 554-557.

11. Joob B, Wiwanitkit V. Ebola outbreak in west Africa. Afr Health Sci. 2014;14(4):1085.

12. Barry M, Traoré FA, Sako FB, Kpamy DO, Bah EI, Poncin M, et al. Ebola outbreak in Conakry, Guinea: epidemiological, clinical, and outcome features. Med Mal Infect. 2014; 44(11-12): 491-494.

13. Green A. West Africa struggles to contain Ebola outbreak. Lancet. 2014; 383(9924): 1196.

14. Victory KR, Coronado F, Ifono SO, Soropogui T, Dahl BA; Centers for Disease Control and Prevention (CDC). Ebola transmission linked to a single traditional funeral ceremony - Kissidougou, Guinea, December, 2014-January 2015. MMWR Morb Mortal Wkly Rep. 2015; 64(14): 386-388.

15. Shrivastava SR, Shrivastava PS, Ramasamy J. Preventing the emergence of Ebola disease in unaffected coun- tries: necessity of preparedness. Int J Health Policy Manag. 2014; 3(7): 417-418.

16. Gulland A. Fifteen countries are at risk of Ebola outbreak, says WHO. BMJ. 2014; 349: g6305.

17. World Health Organization. WHO response to the Ebola Interim Assessment Panel report; 2015. Available from: http://who.int/mediacentre/news/statements/2015/ebola-panel-report/en/

18. Alwan A. The work of WHO in the region: a review of 2014. East Mediterr Health J. 2015; 20(12): 759-760.

19. World Health Organization. Ebola response in action. Geneva: WHO press; 2015.

20. World Health Organization. Contact tracing during an outbreak of Ebola virus disease: Disease surveillance and response programme area disease prevention and control cluster. Republic of Congo: WHO press; 2014.

21. Shrivastava SR, Shrivastava PS, Ramasamy J. Utility of contact tracing in reducing the magnitude of Ebola disease. Germs. 2014; 4(4): 97-99.

22. WHO, CDC. Implementation and management of contact tracing for Ebola virus disease. Geneva: WHO press; 2015.

23. Dallatomasina S, Crestani R, Sylvester Squire J, Declerk H, Caleo GM, Wolz A, et al. Ebola outbreak in rural West Africa: epidemiology, clinical features and outcomes. Trop Med Int Health. 2015; 20(4): 448-454.

24. Blackley DJ, Lindblade KA, Kateh F, Broyles LN, Westercamp M, Neatherlin JC, et al. Rapid intervention to reduce Ebola transmission in a remote village - GbarpoluCounty, Liberia, 2014. MMWR Morb Mortal Wkly Rep. 2015; 64(7): 175-178.

25. Nyenswah T, Blackley DJ, Freeman T, Lindblade KA, Arzoaquoi SK, Mott JA, et al. Community quarantine to interrupt Ebola virus transmission - Mawah Village, Bong County, Liberia, August-October, 2014. MMWR Morb Mortal Wkly Rep. 2015; 64(7): 179-182.

26. Gilsdorf A, Morgan D, Leitmeyer K. Guidance for contact tracing of cases of Lassa fever, Ebola or Marburg haemorrhagic fever on an airplane: results of a European expert consultation. BMC Public Health. 2012; 12: 1014.

27. Lopaz MA, Amela C, Ordobas M, Dominguez-Berjon MF, Alvarez C, Martinez M, et al. First secondary case of Ebola outside Africa: epidemiological characteristics and contact monitoring, Spain, September to November 2014. Euro Surveill. 2015; 20(1): 21003.

28. Smith CL, Hughes SM, Karwowski MP, Chevalier MS, Hall E, Joyner SN, et al. Addressing needs of contacts of Ebola patients during an investigation of an Ebola cluster 
in the United States - Dallas, Texas, 2014. MMWR Morb Mortal Wkely Rep. 2015; 64(5): 121-123.

29. Nyenswah T, Fallah M, Sieh S, Kollie K, Badio M, Gray A, et al. Controlling the last known cluster of Ebola virus disease - Liberia, January-February 2015. MMWR Morb Mortal Wkely Rep. 2015; 64(18): 500-504.

30. Kaasik-Aaslav K, Coulombier D. The tail of the epidemic and the challenge of tracing the very last Ebola case. Euro Surveill. 2015; 20(12): 21075.

31. Hagan JE, Smith W, Pillai SK, Yeoman K, Gupta S, Neatherlin J, et al. Implementation of Ebola case-finding using a village chieftaincy taskforce in a remote outbreak -Liberia, 2014. MMWR Morb Mortal Wkly Rep. 2015; 64(7): 183-185.

32. Wells C, Yamin D, Ndeffo-Mbah ML, Wenzel N, Gaffney SG, Townsend JP, et al. Harnessing case isolation and ring vaccination to control Ebola. PLoS Negl Trop Dis. 2015; 9(5): e0003794.

33. Browne C, Gulbudak H, Webb G. Modeling contact tracing in outbreaks with application to Ebola. $J$ Theor Biol. 2015; 384: 33-49.

34. Shultz JM, Baingana F, Neria Y. The 2014 Ebola outbreak and mental health: current status and recommended response. JAMA. 2015; 313(6): 567-568.

35. Shrivastava SR, Shrivastava PS, Ramasamy J. Exploring the importance of infection prevention and control measures in the 2014 outbreak of Ebola. Int J Prev Med. 2015; 6(10): 96.

36. Logan G, Vora NM, Nyensuah TG, Gasasira A, Mott J, Walke H, et al. Establishment of a community care center for isolation and management ofEbola patients -Bomi County, Liberia, October 2014. MMWR Morb Mortal Wkly Rep. 2014; 63(44): 1010-1012.

37. Shrivastava SR, Shrivastava PS, Ramasamy J. Insights from the Ebola virus disease outbreak. Ann Afr Med. 2015; 14(4): 200-201.

38. Tracey LE, Regan AK, Armstrong PK, Dowse GK, Effler PV. EbolaTracks: an automated SMS system for monitoring persons potentially exposed to Ebola virus disease. Euro Surveill. 2015; 20(1): 20999.

39. Boozary AS, Farmer PE, Jha AK. The Ebola outbreak, fragile health systems, and quality as a cure. JAMA. 2014; 312(18): 1859-1860.

40. Gesser-Edelsburg A, Shir-Raz Y, Hayek S, Sassoni-Bar Lev O. What does the public know about Ebola? The public's risk perceptions regarding the current Ebola outbreak in an as-yet unaffected country. Am J Infect Control. 2015; 43(7): 669-675.

African Health Sciences Vol 17 Issue 1, March, 2017
41. Goldstone BJ, Brown B. The role of public knowledge, resources, and innovation inresponding to the Ebola outbreak. Disaster Med Public Health Prep. 2015; 9(5): 595-597.

42. Kinsman J. "A time of fear": local, national, and international responses to a large Ebola outbreak in Uganda. Global Health. 2012; 8: 15.

43. Chung WM, Smith JC, Weil LM, Hughes SM, Joyner SN, Hall EM, et al. Active tracing and monitoring of contacts associated with the first cluster of Ebola in the United States. Ann Intern Med. 2015; 163(3): 164-173.

44. Shrivastava SR, Shrivastava PS, Ramasamy J. Ebola epidemic in West Africa: Identified challenges and recommended measures. Saudi J Health Sci. 2014; 4(2): 141142.

45. World Health Organization. Communication for behavioural impact (COMBI): A toolkit for behavioural and social communication in outbreak response. Geneva: WHO press; 2012.

46. Nsubuga P. The Ebola outbreak in West Africa: a story of related public health challenges and a pointer to solutions to mitigate the inevitable next outbreak. Pan AfrMed J. 2014; 19: 48.

47. Gulland A. More health staff are needed to contain Ebola outbreak, warns WHO. BMJ. 2014; 349: g5485.

48. Pandey A, Atkins KE, Medlock J, Wenzel N, Townsend JP, Childs JE, et al. Strategies for containing Ebola in West Africa. Science. 2014; 346(6212): 991-995.

49. World Health Organization. Persistent virus in people recovering from Ebola virus disease; 2015. Available from: http://who.int/csr/disease/ebola/virus-persistence/en/

50. World Health Organization. WHO congratulates Senegal on ending Ebola transmission; 2014. Available from:http://www.who.int/mediacentre/news/statements/2014/senegal-ends-ebola/en/

51. World Health Organization. WHO declares end of Ebola outbreak in Nigeria; 2014. Available from: http:// who.int/mediacentre/news/statements/2014/nigeria-ends-ebola/en/

52. Baştuğ A, Bodur H. Ebola viral disease: What should be done to combat the epidemic in 2014? Turk J Med Sci. 2015; 45(1): 1-5.

53. Lamunu M, Lutwama JJ, Kamugisha J, Opio A, Nambooze J, Ndayimirije N, et al. Containing a haemorrhagic fever epidemic: the Ebola experience in Uganda (October 2000-January 2001). Int J Infect Dis. 2004; 8(1): 27-37. 54. Shrivastava SR, Shrivastava PS, Ramasamy J. Lessons 
learnt from the 2014 Ebola outbreak in West-Africa. J Res Med Sci. 2015; 20(1): 107-108.

55. Parsons C, Naeem Ahmad U. The West African Ebola outbreak: finishing the job, preparing for future. Trans R Soc Trop Med Hyg. 2015; 109(8): 481-482.

56. Patel U, Pharr JR, Ihesiaba C, Oduenyi FU, Hunt AT, Patel D, et al. Ebola outbreak in Nigeria: Increasing ebola knowledge of volunteer health advisors. Glob J Health Sci. 2015; 8(1): 46199.

57. Shrivastava SR, Shrivastava PS, Ramasamy J. Public health strategies to ensure optimal community participation in the Ebola outbreak in West-Africa. J Res Med Sci. 2015; 20(3): 318-319.

58. Chen Y, Liu C, Yang R. The coordination of international organizations and its role in the prevention and control of ebola outbreak in Sierra Leone. Zhonghua Yu Fang Yi Xue Za Zbi. 2015; 49(5): 450-451.
59. Reina-Ortiz M, Hoare I, Sharma V, Izurieta R. State of the globe: Ebola outbreak in the western world: Are we really ready? J Glob Infect Dis. 2015; 7(2): 53-55.

60. The Lancet Infectious Diseases. Rationality and coordination for Ebola outbreak in west Africa. Lancet Infect Dis. 2014; 14(12): 1163.

61. Matua GA, Wal DM. Living under the constant threat of Ebola: A phenomenological study of survivors and family caregivers during an Ebola outbreak. J Nurs Res. 2015; 23(3): 217-224.

62. Lushniak BD. Update on the U.S. public health response to the Ebola outbreak. Public Health Rep. 2015; 130(2): 118-120.

63. Wiwanitkit V, Tambo E, Ugwu EC, Ngogang JY, Zhou XN. Are surveillance response systems enough to effectively combat and contain the Ebola outbreak? Infect Dis Poverty. 2015; 4(1): 7. 